\title{
Contribution of germline deleterious variants in the RAD51 paralogs to breast and ovarian cancers
}

\author{
Lisa Golmard ${ }^{1,2} \cdot$ Laurent Castéra $^{3,4} \cdot$ Sophie Krieger $^{3,4,5} \cdot$ Virginie Moncoutier $^{1} \cdot$ Khadija Abidallah $^{1}$. \\ Henrique Tenreiro $^{1} \cdot$ Anthony Laugé $^{1} \cdot$ Julien Tarabeux $^{1,2} \cdot$ Gael A. Millot $^{6,7} \cdot$ André Nicolas $^{1} \cdot$ Marick Laé $^{1}$. \\ Caroline Abadie $^{8}$ - Pascaline Berthet ${ }^{9}$ - Florence Polycarpe ${ }^{9}$ Thierry Frébourg ${ }^{4,10,11}$ - Camille Elan ${ }^{1}$. \\ Antoine de Pauw ${ }^{1} \cdot$ Marion Gauthier-Villars ${ }^{1} \cdot$ Bruno Buecher $^{1} \cdot$ Marc-Henri Stern $\mathbb{D}^{1,2,12}$ •

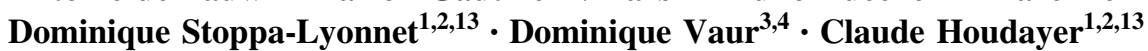

Received: 5 June 2017 / Revised: 20 September 2017 / Accepted: 22 September 2017 / Published online: 8 November 2017

(C) European Society of Human Genetics 2017

\begin{abstract}
$R A D 51$ paralogs $(R A D 51 B, R A D 51 C, R A D 51 D, X R C C 2$, and XRCC3) have recently been involved in breast and ovarian cancer predisposition: $R A D 51 B, R A D 51 C$, and $R A D 51 D$ in ovarian cancer, $R A D 51 B$ and $X R C C 2$ in breast cancer. The aim of this study was to estimate the contribution of deleterious variants in the five $R A D 51$ paralogs to breast and ovarian cancers. The five $R A D 51$ paralog genes were analyzed by next-generation sequencing technologies in germline DNA from 2649 consecutive patients diagnosed with breast and/or ovarian cancer. Twenty-one different deleterious variants were identified in the RAD51 paralogs in 30 patients: $R A D 51 B(n=4), R A D 51 C(n=12), R A D 51 D(n=7), X R C C 2(n=2)$, and XRCC3 $(n=5)$. The overall deleterious variant rate was $1.13 \%$ (95\% confidence interval (CI): $0.72-1.55 \%)$ (30/2649), including 15 variants in breast cancer only cases (15/2063; 0.73\% (95\% CI: 0.34-1.11\%)) and 15 variants in cases with at least one ovarian cancer (15/ $570 ; 2.63 \%$ (95\% CI: $1.24-4.02 \%)$ ). This study is the first evaluation of the five RAD51 paralogs in breast and ovarian cancer predisposition and it demonstrates that deleterious variants can be present in breast cancer only cases. Moreover, this is the first time that XRCC3 deleterious variants have been identified in breast and ovarian cancer cases.
\end{abstract}

\section{Introduction}

Most currently known breast and ovarian cancer predisposition genes play a role in repair of DNA double-strand

Electronic supplementary material The online version of this article (https://doi.org/10.1038/s41431-017-0021-2) contains supplementary material, which is available to authorized users.

$\triangle$ Lisa Golmard

lisa.golmard@curie.fr

1 Department of Tumour Biology, Institut Curie, Paris 75005, France

2 Inserm U830, Institut Curie, Paris 75005, France

3 Department of Cancer Biology and Genetics, CCC François Baclesse, Caen 14000, France

4 Inserm U1079, Normandy Center for Genomic and Personalized Medicine, Rouen 76183, France

5 University of Caen, Normandie 14032, France

6 Institut Curie, PSL Research University, CNRS UMR3244, Paris 75005, France breaks by homologous recombination (HR): BRCAl and $B R C A 2$ are the two major genes and confer high risks of breast and ovarian cancer [1]; PALB2 confers a breast cancer risk modulated by family history and a moderate risk of ovarian cancer [2, 3]; BRIP1 may confer a moderate risk of ovarian cancer only [4].

While breast or ovarian cancer predisposition is caused by monoallelic germline deleterious variants in these genes,

7 Sorbonne Universités, UPMC Univ Paris 06, CNRS UMR3244, Paris 75005, France

8 Department of Genetics, Centre Hospitalo-Universitaire, Rennes 35033, France

9 Department of Genetics, Centre François Baclesse, Caen 14000, France

10 Department of Genetics, Centre Hospitalo-Universitaire, Rouen 76000, France

11 University of Rouen, IRIB, Rouen 76183, France

12 Institut Curie, PSL Research University, Paris 75005, France

13 Paris Descartes University, Sorbonne Paris Cité, Paris 75006, France 
biallelic germline deleterious variants in BRCA2, PALB2, and BRIP1 result in Fanconi anemia, an autosomal recessive inherited syndrome characterized by developmental abnormalities, bone marrow failure and predisposition to various cancers [5]. Rare biallelic germline deleterious variants in BRCAl can result in a Fanconi anemia-like disorder. BRCA2, PALB2, BRIP1, and BRCA1 are called FANCDI, FANCN, FANCJ, and FANCS, respectively, in the context of Fanconi anemia.

Genetic studies were recently conducted on RAD51 paralogs (RAD51B, RAD51C, RAD51D, XRCC2, XRCC3), involved in the same DNA repair pathway: RAD51 is the key protein for HR; BRCA2 loads RAD51 monomers at DNA double-strand break sites and RAD51 activity depends on the RAD51 paralog family [6]. Biallelic germline deleterious variants in $R A D 51 C$ and $X R C C 2$ were identified in patients affected with a Fanconi anemia-like disorder. RAD51C and XRCC2 are called FANCO and $F A N C U$ in the context of Fanconi anemia. As several Fanconi anemia-related genes are also breast and/or ovarian cancer predisposition genes, $R A D 51 C$ was subsequently studied as a candidate gene and was the first $R A D 51$ paralog involved in breast and ovarian cancer predisposition [7].

Monoallelic germline deleterious variants in several RAD51 paralogs have been involved in breast and ovarian cancer predisposition. The strongest evidence comes from identification of monoallelic germline deleterious variants in $R A D 51 C$ and $R A D 51 D$ that confer predisposition to ovarian cancer; their contribution to breast cancer is controversial [7-9]. Monoallelic germline $R A D 51 B$ deleterious variants were reported in a breast and ovarian cancer family case and two unselected cases of ovarian cancer [10, 11]. Monoallelic germline $X R C C 2$ deleterious variants were identified in breast cancer family cases but two subsequent population-based studies failed to confirm an association between $X R C C 2$ deleterious variants and breast cancer risk [12-14]. Finally, no XRCC3 deleterious variant was identified in breast and ovarian cancer cases but some XRCC3 neutral variants were associated with breast and ovarian cancer susceptibility $[15,16]$.

In this study, the five RAD51 paralogs were analyzed on a large series of consecutive unrelated patients to better estimate their contribution to breast and ovarian cancers.

\section{Patients and methods}

\section{Patients}

This study was conducted on a series of 2649 consecutive unrelated patients diagnosed with breast and/or ovarian cancer, including 2063 patients with personal and family history of breast cancer only, 570 patients with at least 1 ovarian cancer in their personal or family history, 9 patients with personal or family history of pancreas cancer and 7 patients with personal or family history of prostate cancer. Genetic testing for the RAD51 paralogs was proposed to patients based on personal or family history, in addition to BRCAI/2 genetic testing. Individual inclusion criteria were: (1) breast adenocarcinoma before the age of 36 , (2) nonmucinous ovarian carcinoma before the age of 70 , (3) triple-negative breast adenocarcinoma before the age of 51, (4) adenocarcinoma with medullary features, (5) breast and ovarian carcinomas, or (6) male breast cancer. Family history was defined as either (1) three breast cancer cases in first-degree or second-degree relatives in the same lineage, (2) two breast cancer cases in first-degree or second-degree relatives (with a transmitting male), with one cancer before the age of 40 or one cancer before 50 and the other before 70 , or (3) one breast cancer case and one first-degree or second-degree relative (with a transmitting male) with ovarian cancer. Family history was the unique inclusion criterion for 112 patients that were unaffected by breast or ovarian cancer. All patients attended a visit for genetic counseling in a family cancer clinic. Patients gave their informed consent for genetic testing.

\section{Genomic DNA analysis}

Two different protocols of next-generation sequencing (NGS) were used for gene analysis of RAD51 paralog coding exons and exon-intron junctions. Gene analysis was performed by SureSelect ${ }^{\mathrm{XT}}$ (Agilent) enrichment and sequencing on GAIIx (Illumina) for 1701 patients, as previously described [17], or AmpliSeq (Life Technologies) enrichment and sequencing on Personal Genome Machine (PGM, Life Technologies), followed by bioinformatics analysis using the NextGENe software v2.3 (SoftGenetics), for 948 patients. AmpliSeq enrichment was performed on pools of 20 patient DNA for higher throughput instead of individual analysis.

\section{Variant classification criteria}

Criteria for deleterious variant class (variants that affect function) were: nonsense substitutions, frameshift insertions/deletions, or splicing variants leading to out-of-frame exon skipping or in-frame exon skipping located in a functional domain, confirmed by mRNA analysis. This class corresponds to pathogenic variants according to recommendations from the American College of Medical Genetics (ACMG), except RAD51B p.(Arg8*) and p.(Arg47*) that would be considered as likely pathogenic as they were reported in population databases in two or one control, respectively, and $R A D 51 C$ c.706-2A $>\mathrm{G}$ and $\mathrm{A}>\mathrm{T}$, likely pathogenic as they are not null variants but lead to inframe exon skipping in a functional domain (Table 1) [18]. 


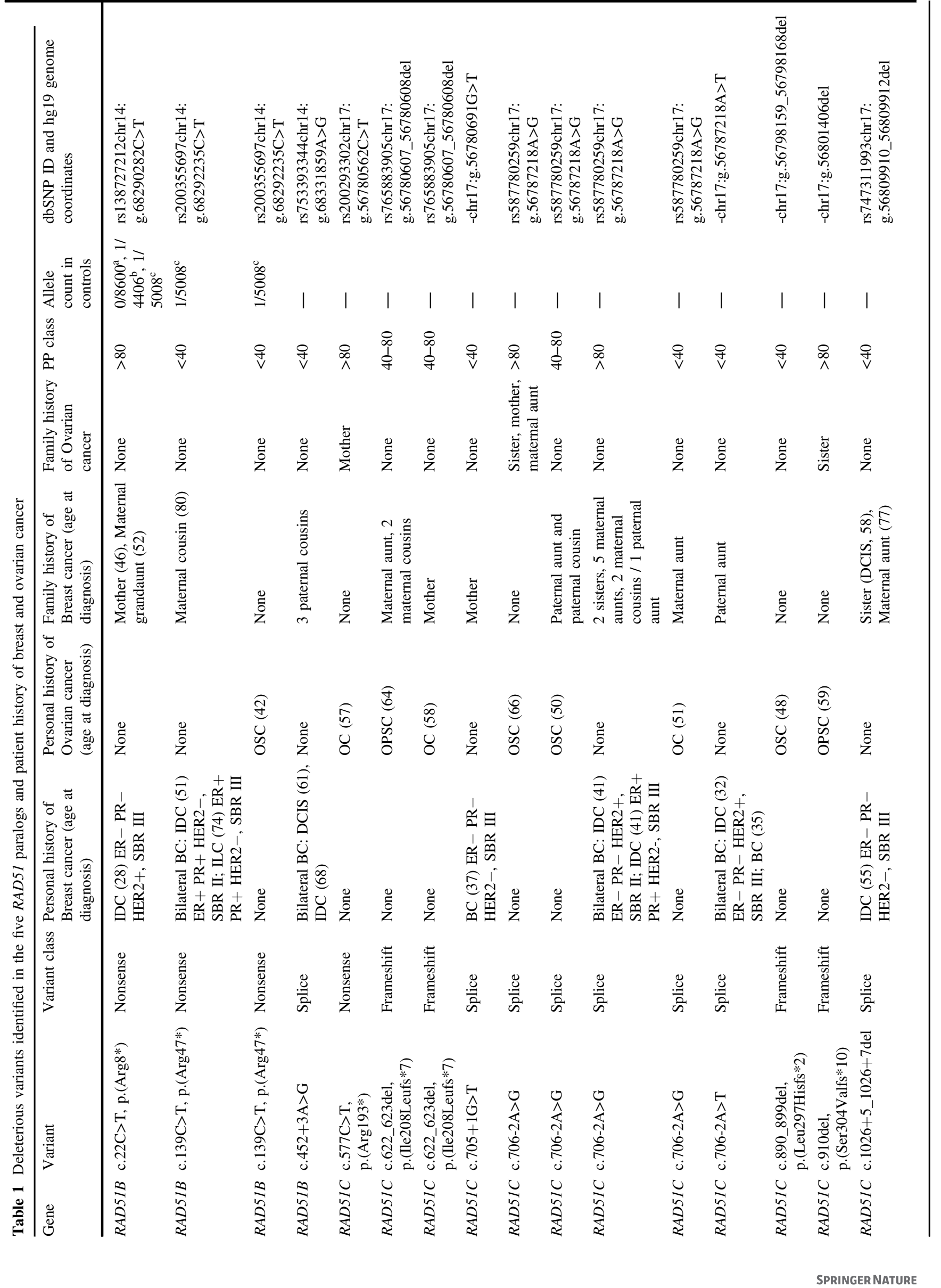




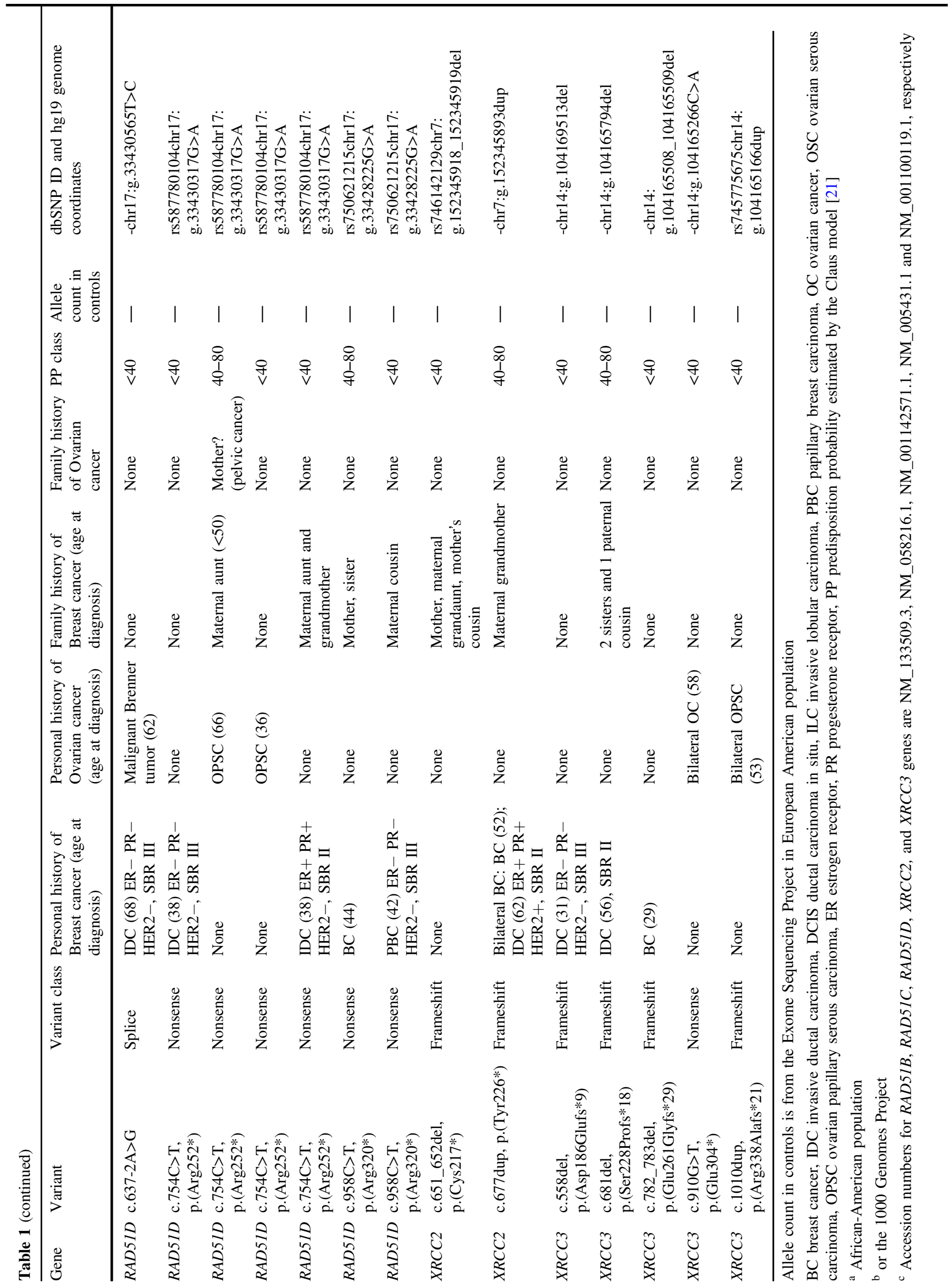


Criteria for likely deleterious variant class (variants that probably affect function) were: splicing variants by in silico prediction, missense variants with Align-GVGD class ranging from C45 to C65 [19], in-frame insertions/deletions, or stop-loss variants. This class corresponds to variants of unknown significance according to ACMG recommendations. The splicing effect of variants was predicted according to a previously published bioinformatics pipeline: a greater than $15 \%$ decrease of the MaxEntScan score and a greater than 5\% decrease of the SpliceSiteFinder-like score for donor/acceptor splice sites [20].

\section{Variant annotation}

Accession numbers used in this report for $R A D 51 B$, $R A D 51 C, R A D 51 D, X R C C 2$ and XRCC3 genes were NM_133509.3, NM_058216.1, NM_001142571.1, NM_005431.1, and NM_001100119.1, respectively. Variants were submitted to LOVD databases, at https://databa ses.lovd.nl/shared/genes/RAD51B, RAD51C, RAD51D, XRCC2, or XRCC3.

\section{mRNA analysis}

RNA was extracted from breast tumors using TRIzol reagent according to the manufacturer's instructions (Invitrogen). $2 \mu \mathrm{g}$ of total RNA from each sample was used for reverse transcription in a $40 \mu \mathrm{L}$ reaction using the GeneAmp RNA PCR Core kit according to the manufacturer's instructions (Applied Biosystems). cDNA was amplified with forward and reverse primers 5'-TGCACAACTTCAAGGCAATC- $3^{\prime}$ and $5^{\prime}$-TTGGGTGACAGAGCAAAATG- $3^{\prime}$ for $R A D 51 B$ c. $1036+5 \mathrm{G}>\mathrm{A}$ variant, 5'-TGACCTGTCTCTTCGTACTCG- $3^{\prime}$ and $5^{\prime}$-TCCACTTGTACACATTGATTTCAC- $3^{\prime}$ for $R A D 51 C$ c. $1026+5 \_1026+7$ del variant.

\section{Results}

\section{Genetic variants in $R A D 51$ paralogs}

Twenty-one different deleterious variants were identified in the $R A D 51$ paralogs in 30 patients: $R A D 51 B(n=4)$, $R A D 51 C(n=12), \operatorname{RAD51D}(n=7), \operatorname{XRCC2}(n=2)$ and XRCC3 $(n=5)$ (Table 1) [21]. The overall deleterious variant rate was $1.13 \%$ (95\% confidence interval (CI): $0.72-1.55 \%)(30 / 2649)$. The deleterious variant classes were nonsense $(n=11 ; 37 \%)$, frameshift $(n=10 ; 33 \%)$ or splice $(n=9 ; 30 \%)$. RAD51C c.706-2 $\mathrm{A}>\mathrm{G}$ and $R A D 51 D$ p.(Arg252*) variants were observed in four unrelated patients. In addition, 15 likely deleterious variants were identified in 22 patients, predominantly missense variants (Supplementary Table 1). These variants were not taken into account in the contribution to breast and ovarian cancers as their causality needs to be assessed. Among them, $R A D 51 B$ c. $1036+5 \mathrm{G}>\mathrm{A}$ variant was detected in four unrelated patients; its impact on splicing was confirmed by mRNA analysis but this variant was not included because of its frequency in controls (4/2649; $0.15 \%$ patients vs. 5/ 8600; $0.06 \%$ controls from European American population in the Exome Sequencing Project; $p=0.28$ ).

\section{Clinicopathological characteristics of breast and ovarian cancers in RAD51 paralog deleterious variant carriers}

Patients mutated in a RAD51 paralog gene were diagnosed with breast cancer $(n=15)$, ovarian cancer $(n=13)$ or both breast and ovarian cancer $(n=1)$. One patient was unaffected and included for breast cancer family history only (Table 1).

Among the 15 patients diagnosed with breast cancer, 5 were bilateral cases. The overall mean age of onset at first diagnosis of breast cancer was 45 years (range 27-68) (Table 2). The histological type of breast cancer was mostly invasive ductal carcinoma (IDC) for the five RAD51 paralogs, but the histological subtypes were heterogeneous: the most frequent subtype was triple negative (estrogen and progesterone receptor-negative, HER2-negative (ER-, PR-, HER2-)) (6/14), which was observed for two RAD51C (2/5), three RAD51D (3/4) and one XRCC3-mutated (1/1) breast carcinomas but not observed in three $R A D 51 B$ and one $X R C C 2$-mutated breast carcinomas. RAD51B-mutated breast carcinomas were predominantly hormone receptor-positive $(\mathrm{ER}+, \mathrm{PR}+)$ and HER2-negative (2/3).

Among the 13 patients diagnosed with ovarian cancer, the overall mean age of onset at first diagnosis was 55 years (range 36-66) (Table 2). The histological type of ovarian cancer was mostly serous carcinoma. A rare type of ovarian cancer was observed, a malignant Brenner tumor, in a patient carrying a $R A D 51 D$ deleterious variant.

Table 2 Summary of age at diagnosis for breast and ovarian cancer for $R A D 51$ paralog deleterious variant carriers

\begin{tabular}{lcccccc}
\hline Gene & $R A D 51 B$ & $R A D 51 C$ & $R A D 51 D$ & $X R C C 2$ & $X R C C 3$ & Total \\
\hline \multicolumn{2}{l}{ Breast cancer } & & & & & \\
$n$ & 3 & 4 & 5 & 1 & 3 & 20 \\
Range & $28-61$ & $32-55$ & $38-68$ & $52-52$ & $29-56$ & $27-68$ \\
Median & 51 & 39 & 42 & 52 & 31 & 43 \\
Mean & 47 & 41 & 46 & 52 & 39 & 45 \\
Ovarian cancer & & & & & \\
$n$ & 1 & 8 & 3 & 0 & 2 & 14 \\
Range & $42-42$ & $48-66$ & $36-66$ & & $53-58$ & $36-66$ \\
Median & 42 & 58 & 62 & & 56 & 58 \\
Mean & 42 & 57 & 55 & & 56 & 55 \\
\hline
\end{tabular}

Age at first diagnosis only was considered for bilateral cases 


\section{Personal and family history of breast and ovarian cancer}

Among the 30 patients with RAD51 paralog deleterious variants, 15 variants were identified in breast cancer only cases (15/2063; $0.73 \%$ (95\% CI: $0.34-1.11 \%)$ ) and 15 variants in cases with at least one ovarian cancer in their personal or family history $(15 / 570 ; 2.63 \%(95 \% \mathrm{CI}$ : 1.24-4.02\%)) (Table 3). Concerning breast cancer only cases, deleterious variants were identified in the five $R A D 51$ paralogs, with the highest rate in RAD51D (4 deleterious variants; $0.19 \%$ ). Regarding cases with at least one ovarian cancer, $X R C C 2$ was the only gene with no detected deleterious variant; the highest rate was in $R A D 51 C$ (9 deleterious variants; $1.58 \%$ ).

\section{Discussion}

This study evaluated the contribution of germline deleterious variants in the five $R A D 51$ paralogs to breast and ovarian cancers. These variants were detected at an overall rate of $1.13 \%$ [95\% CI: $0.72-1.55 \%$ ], in breast cancer only cases $(0.73 \%(95 \% \mathrm{CI}: 0.34-1.11 \%))$ or cases with at least one ovarian cancer (2.63\% (95\% CI: $1.24-4.02 \%)$ ).

\section{$R A D 51$ paralog deleterious variant rate}

RAD51 paralog deleterious variant rate may be underestimated as variants that were likely deleterious by in silico prediction were also identified, in 22 patients (22/2649; $0.83 \%$ (95\% CI: 0.47-1.19\%)). The overall deleterious variant rate could therefore range from $1.13 \%$ (95\% CI: $0.72-1.55 \%$ ) to $1.96 \%$ (95\% CI: $1.43-2.50 \%$ ). Functional assays are needed to estimate more accurately the contribution of germline $R A D 51$ paralog deleterious variants to

Table 3 Distribution of RAD51 paralog deleterious variants in breast or ovarian cancer

\begin{tabular}{llllll}
\hline \multirow{2}{*}{ Gene } & \multicolumn{3}{l}{ At least one Ovarian cancer } & \\
\cline { 3 - 5 } & $\begin{array}{l}\text { Breast } \\
\text { cancer } \\
\text { only }\end{array}$ & $\begin{array}{l}\text { Breast and } \\
\text { ovarian }\end{array}$ & $\begin{array}{l}\text { Ovarian } \\
\text { cancer } \\
\text { oncer }\end{array}$ & $\begin{array}{l}\text { Total for } \\
\text { ovarian } \\
\text { cancer }\end{array}$ & \\
& $n=2063$ & $n=538$ & $n=32$ & $n=570$ & $n=2649$ \\
& $n(\%)$ & $n(\%)$ & $n(\%)$ & $n(\%)$ & $n(\%)$ \\
\hline$R A D 51 B$ & $3(0.15)$ & $0(0.00)$ & $1(3.13)$ & $1(0.18)$ & $4(0.15)$ \\
$R A D 51 C$ & $3(0.15)$ & $8(1.49)$ & $1(3.13)$ & $9(1.58)$ & $12(0.45)$ \\
RAD51D & $4(0.19)$ & $2(0.37)$ & $1(3.13)$ & $3(0.53)$ & $7(0.26)$ \\
XRCC2 & $2(0.10)$ & $0(0.00)$ & $0(0.00)$ & $0(0.00)$ & $2(0.08)$ \\
XRCC3 & $3(0.15)$ & $0(0.00)$ & $2(6.25)$ & $2(0.35)$ & $5(0.19)$ \\
Total & $15(0.73)$ & $10(1.86)$ & $5(15.6)$ & $15(2.63)$ & $30(1.13)$ \\
\hline
\end{tabular}

breast and ovarian cancers. As each RAD51 paralog is necessary for HR, these assays could be measurement of HR frequency by DR-GFP or cell sensitivity to poly-(ADPribose) polymerase (PARP) inhibitors, by cDNA-based complementation approach in cells deficient for the tested RAD51 paralog. Indeed, DR-GFP assay has been previously published for the five $R A D 51$ paralogs and cell sensitivity to PARP inhibitors for $R A D 51 C, R A D 51 D$, and XRCC2 [8, 22-25].

$R A D 51$ paralog deleterious variants were identified in patients negative for $B R C A 1 / 2$ deleterious variants but one patient was double heterozygote for a XRCC2 likely deleterious variant and a $B R C A 1$ deleterious variant (Supplementary Table 1). Co-occurrence of RAD51C and BRCA2 deleterious variants has been previously reported in a breast cancer family [26].

\section{Clinicopathological characteristics of breast and ovarian} cancers in $R A D 51$ paralog deleterious variant carriers

The most frequent histological type of breast cancer was IDC, as in the general population and previously reported for $R A D 51 D$-mutated breast tumors [14]. The histological subtypes of breast tumors were heterogeneous, as it was described in two previous reports on $R A D 51 C$-mutated tumors that suggested these tumors were similar to $B R C A 2$ mutated breast tumors [13, 27]. Heterogeneity of histological subtypes was also reported for $R A D 51 B$ in a study conducted on 46,036 invasive breast cancer cases and 46,930 controls that observed an association between $R A D 51 B$ rs10483813 and rs999737 SNPs and breast cancer for most tumor subtypes [28]. The most frequent subtype was triple-negative, which was observed for $R A D 51 C$, $R A D 51 D$ and $X R C C 2$. This result is consistent with a recent study of over 35,000 women with breast cancer tested with a 25-gene panel of hereditary cancer genes, which revealed that the prevalence of deleterious variants in $R A D 51 C$ was statistically higher among women with triple-negative breast cancer [29].

The most frequent histological type of ovarian cancer was serous carcinoma, as in the general population and in BRCA1/2-mutated tumors [30]. This result was also previously reported for $R A D 51 B, R A D 51 C$, and $R A D 51 D$ ovarian tumors [8, 11, 31].

\section{Personal and family history of breast and ovarian cancer}

$R A D 51 B$

The $R A D 51 B$ deleterious variant rate was $0.15 \%$, with three variants identified in breast cancer only cases (3/2063; $0.15 \%$ ) and only one variant among cases with at least one 
ovarian cancer $(1 / 570 ; 0.18 \%)$. To our knowledge, only one $R A D 51 B$ deleterious variant was reported in a breast cancer case with an ovarian cancer family history [10]. A recent case-control study conducted on unselected ovarian cancer cases observed a low $R A D 51 B$ deleterious variant rate at $0.06 \%(2 / 3401)$ in cases and no deleterious variant in 2769 controls [11]. Numerous Genome-Wide Association Studies (GWAS) or case-control studies identified several RAD51B neutral variants as susceptibility factors for breast cancer [27, 28, 32-35]. Overall, these data suggest that $R A D 51 B$ is involved in breast cancer predisposition but further studies are needed to evaluate its contribution to ovarian cancer.

\section{RAD51C}

The RAD51C deleterious variant rate was $0.45 \%$, with three variants identified in breast cancer only cases (3/2063; $0.15 \%$ ) and nine variants among cases with at least one ovarian cancer $(9 / 570 ; 1.58 \%)$. RAD51C was the predominant $R A D 51$ paralog with deleterious variants identified in cases with at least one ovarian cancer, and the rate of $1.58 \%$ is quite similar to the rate of $1.3 \%$ previously reported for $R A D 51 C$ germline deleterious variants in breast and ovarian cancer cases [7, 36]. RAD51C contribution to ovarian cancer has been established by numerous studies but its contribution to breast cancer is less clear [9, 36-38]. Indeed, the first study by Meindl et al. identified 6 RAD51C deleterious variants in 480 cases with breast and ovarian cancer but no deleterious variant in 620 breast cancer only cases. Similar results were observed in other studies [9, 38]. Loveday et al. estimated the relative risk (RR) of ovarian cancer for $R A D 51 C$ deleterious variant carriers to 5.88, with no evidence of breast cancer association [9]. A recent casecontrol study on unselected ovarian cancer cases estimated the odds ratio for $R A D 51 C$ deleterious variants to be 5.2 [11]. The $R A D 51 C$ deleterious variant rate in unselected ovarian cases was lower $(0.41 \%)$. However, three RAD $51 C$ deleterious variants were reported in breast cancer only cases[39-41]. Taking these results together with our results, we estimate that $R A D 51 C$ contribution to breast cancer predisposition should be considered.

\section{$R A D 51 D$}

The RAD51D deleterious variant rate was $0.26 \%$, with four variants identified in breast cancer only cases (4/2063; $0.19 \%$ ) and three variants among cases with at least one ovarian cancer $(3 / 570 ; 0.53 \%)$. This $R A D 51 D$ deleterious variant rate of $0.53 \%$ in cases with at least one ovarian cancer is lower than the first study that established RAD51D as an ovarian cancer predisposition gene, with a deleterious variant rate of $0.9 \%$ (8/911) [8]. This discrepancy may be explained by a higher number of ovarian cancer cases in families studied by Loveday et al., as they reported a higher association with ovarian cancer for families with three or more affected individuals. Like $R A D 51 C$, contribution of $R A D 51 D$ germline deleterious variants to ovarian cancer has been established by several studies but their contribution to breast cancer is less clear. In the first report on $R A D 51 D$, the relative risk of ovarian cancer for $R A D 51 D$ deleterious variants was estimated to be 6.30 whereas the relative risk of breast cancer was 1.32 . RAD51D deleterious variants in breast and ovarian cancer family cases were also observed in subsequent studies [42, 43]. A recent case-control study on unselected ovarian cancer cases estimated the odds ratio of ovarian cancer for $R A D 51 D$ deleterious variants to be 12 , but the $95 \%$ CI was wide (95\% CI: (1.5-90)) [11]. The RAD51D deleterious variant rate in unselected ovarian cancer cases was $0.35 \%$. To our knowledge, only one breast cancer only family case with $R A D 51 D$ deleterious variant was reported [44]. However, presence of numerous $R A D 51 D$ deleterious variant carriers affected with breast cancer was recently reported, albeit in the context of familial ovarian cancer [42]. Taking these results together with our results, we estimate that $R A D 51 D$ contribution to breast cancer predisposition should be considered.

\section{$X R C C 2$}

Two XRCC2 deleterious variants were identified in breast cancer only cases $(2 / 2063 ; 0.10 \%)$ and no deleterious variant in 570 cases with at least one ovarian cancer. These results are consistent with a previous report of $X R C C 2$ deleterious variants in multiple breast cancer cases [12]. However, this association was not confirmed in two subsequent studies, although a relative risk $<2$ could not be excluded $[13,14]$. Several case-control studies evaluated association of XRCC2 p.(Arg188His) neutral variant (rs3218536:G $>$ A) with breast and ovarian cancer. Its association with breast cancer is controversial but its association with ovarian cancer was observed in three meta-analyses[45-47]. Overall, the low $X R C C 2$ deleterious variant rate needs studies on several thousands of cases and controls to evaluate XRCC2 contribution to breast and ovarian cancer.

\section{$X R C C 3$}

The XRCC 3 deleterious variant rate was $0.19 \%$, with three variants identified in breast cancer only cases (3/2063; $0.15 \%)$ and two variants in ovarian cancer only cases $(2 / 32$; $6.25 \%$ ). XRCC3 deleterious variant carriers had the lowest mean age of breast cancer onset, at 39 years. This is the first report of XRCC3 deleterious variants in breast and ovarian cancer cases. Combining these data with case-control studies that observed an association between $X R C C 3$ neutral variants and breast and ovarian cancer suggest that $X R C C 3$ deleterious variants may predispose to breast and ovarian cancer. 


\section{Follow-up strategies}

In this study and previous reports on RAD51 paralogs concerning breast and ovarian cancer predisposition, there is an ascertainment bias for young age of onset as this is an inclusion criterion for genetic testing. However, except the unique $X R C C 2$ deleterious variant carrier diagnosed with breast cancer at 52 years, the mean age of breast cancer onset for any RAD51 paralog deleterious variant carriers, 45 years, was similar to those reported in $B R C A 1$ and $B R C A 2$ deleterious variant carriers [1]. A specific breast cancer follow-up at younger age should therefore be recommended to $R A D 51$ paralog deleterious variant carriers.

Two previous reports concluded that the high risk of ovarian cancer conferred by $R A D 51 C$ germline deleterious variants should lead to suggestion of preventive oophorectomy, before or after menopause in the study by Blanco et al. [39] or Sopik et al. [48], as the mean age of ovarian cancer was estimated to be 49 or 60 , respectively. A recent study proposed premenopausal preventive oophorectomy in $R A D 51 C$ and $R A D 51 D$ deleterious variant carriers as $18 \%$ of ovarian cancers in these patients occurred before 50 years of age [11]. In this study, 1 out of 8 ovarian cancers for $R A D 51 C$ and 1 out of 3 ovarian cancers for RAD51D occurred before 50 years of age, at 48 and 36 years, respectively. The ascertainment bias for young age of onset is lower for ovarian cancer than for breast cancer as the personal history-based inclusion criterion is an ovarian cancer before the age of 70 (vs. before the age of 36 or 51 for breast cancer, for all subtypes or triple-negative breast cancer, respectively) and the family history-based inclusion criterion is an ovarian cancer whatever the age of onset. Given the poor prognosis of ovarian cancer and the elevated relative risks of ovarian cancer, premenopausal preventive oophorectomy should be discussed with $R A D 51 C$ and $R A D 51 D$ deleterious variant carriers.

PARP inhibitors have recently been validated as a new treatment of BRCA1/2-mutated ovarian cancer, and target tumor cells that are defective for DNA repair by HR [49]. As RAD51 paralogs are involved in the same pathway, PARP inhibitors could also be effective on RAD51 paralogmutated ovarian cancer. Some in vitro studies conducted on $R A D 51 C$ and $R A D 51 D$-mutated tumor cells observed a sensitivity to PARP inhibitors, supporting their inclusion in clinical trials $[8,23]$.

\section{Conclusion}

This study is the first evaluation of the five RAD51 paralogs in breast and ovarian cancer predisposition and it demonstrates that deleterious variants can be present in breast cancer only cases. Moreover, this is the first time that
XRCC3 deleterious variants have been identified in breast and ovarian cancer cases. Given the low deleterious variant rate of $R A D 51$ paralogs, further studies are needed to estimate more accurately their clinicopathological characteristics. This study constitutes a sound basis for penetrance risk estimates through the genetic testing of relatives of variant carriers.

Acknowledgements This work was supported by INCa and DGOS in the context of the SiRIC program \#2011-189.

Compliance with ethical standards

Conflict of interest The authors declare no conflict of interest.

\section{References}

1. Mavaddat N, Peock S, Frost D, et al. Cancer risks for BRCA1 and BRCA2 mutation carriers: results from prospective analysis of EMBRACE. J Natl Cancer Inst. 2013;105:812-22.

2. Antoniou AC, Casadei S, Heikkinen T, et al. Breast-cancer risk in families with mutations in PALB2. $\mathrm{N}$ Engl $\mathrm{J}$ Med. 2014;371:497-506.

3. Norquist BM, Harrell MI, Brady MF, et al. Inherited mutations in women with ovarian carcinoma. JAMA Oncol. 2016;2:482-90.

4. Easton DF, Lesueur F, Decker B, et al. No evidence that protein truncating variants in BRIP1 are associated with breast cancer risk: implications for gene panel testing. $\mathrm{J}$ Med Genet. 2016;53:298-309.

5. Bogliolo M, Surrallés J. Fanconi anemia: a model disease for studies on human genetics and advanced therapeutics. Curr Opin Genet Dev. 2015;33:32-40.

6. Prakash R, Zhang Y, Feng W, Jasin M. Homologous recombination and human health: the roles of BRCA1, BRCA2, and associated proteins. Cold Spring Harb Perspect Biol. 2015;7: a016600.

7. Meindl A, Hellebrand $\mathrm{H}$, Wiek C, et al. Germline mutations in breast and ovarian cancer pedigrees establish RAD51C as a human cancer susceptibility gene. Nat Genet. 2010;42:410-14.

8. Loveday C, Turnbull C, Ramsay E, et al. Germline mutations in RAD51D confer susceptibility to ovarian cancer. Nat Genet. 2011;43:879-82.

9. Loveday C, Turnbull C, Ruark E, et al. Germline RAD51C mutations confer susceptibility to ovarian cancer. Nat Genet. 2012;44:475-76. author reply 476

10. Golmard L, Caux-Moncoutier V, Davy G, et al. Germline mutation in the RAD51B gene confers predisposition to breast cancer. BMC Cancer. 2013;13:484.

11. Song H, Dicks E, Ramus SJ, et al. Contribution of germline mutations in the RAD51B, RAD51C, and RAD51D genes to ovarian cancer in the population. J Clin Oncol. 2015;33:2901-7.

12. Park DJ, Lesueur F, Nguyen-Dumont T, et al. Rare mutations in XRCC2 increase the risk of breast cancer. Am J Hum Genet. 2012;90:734-39.

13. Hilbers FS, Wijnen JT, Hoogerbrugge N, et al. Rare variants in XRCC2 as breast cancer susceptibility alleles. J Med Genet. 2012;49:618-20.

14. Pelttari LM, Kiiski JI, Ranta S, et al. RAD51, XRCC3, and $\mathrm{XRCC} 2$ mutation screening in Finnish breast cancer families. SpringerPlus. 2015;4:92.

15. He X-F, Wei W, Su J, et al. Association between the XRCC3 polymorphisms and breast cancer risk: meta-analysis based on case-control studies. Mol Biol Rep. 2012;39:5125-34. 
16. Yuan C, Liu X, Yan S, Wang C, Kong B. Analyzing association of the XRCC3 gene polymorphism with ovarian cancer risk. Biomed Res Int. 2014;2014:648137.

17. Castéra L, Krieger S, Rousselin A, et al. Next-generation sequencing for the diagnosis of hereditary breast and ovarian cancer using genomic capture targeting multiple candidate genes. Eur J Hum Genet. 2014;22:1305-13.

18. Richards S, Aziz N, Bale S, et al. Standards and guidelines for the interpretation of sequence variants: a joint consensus recommendation of the American College of medical genetics and genomics and the association for molecular pathology. Genet Med. 2015;17:405-24.

19. Tavtigian SV, Deffenbaugh AM, Yin L, et al. Comprehensive statistical study of 452 BRCA1 missense substitutions with classification of eight recurrent substitutions as neutral. J Med Genet. 2006;43:295-05.

20. Houdayer C, Caux-Moncoutier V, Krieger S, et al. Guidelines for splicing analysis in molecular diagnosis derived from a set of 327 combined in silico/in vitro studies on BRCA1 and BRCA2 variants. Hum Mutat. 2012;33:1228-38.

21. Claus EB, Risch N, Thompson WD. Genetic analysis of breast cancer in the cancer and steroid hormone study. Am J Hum Genet. 1991;48:232-42.

22. Chun J, Buechelmaier ES, Powell SN. Rad51 paralog complexes BCDX2 and CX3 act at different stages in the BRCA1-BRCA2dependent homologous recombination pathway. Mol Cell Biol. 2013;33:387-95.

23. Min A, Im S-A, Yoon Y-K, et al. RAD51C-deficient cancer cells are highly sensitive to the PARP inhibitor olaparib. Mol Cancer Ther. 2013;12:865-77.

24. Hilbers FS, Luijsterburg MS, Wiegant WW, et al. Functional analysis of missense variants in the putative breast cancer susceptibility gene XRCC2. Hum Mutat. 2016;37:914-25.

25. Rivera B, Di Iorio M, Frankum J, et al. Functionally null RAD51D missense mutation associates strongly with ovarian carcinoma. Cancer Res. 2017;77:4517-29.

26. Ahlborn LB, Steffensen AY, Jønson L, et al. Identification of a breast cancer family double heterozygote for RAD51C and BRCA2 gene mutations. Fam Cancer. 2015;14:129-33.

27. Long J, Zhang B, Signorello LB, et al. Evaluating genome-wide association study-identified breast cancer risk variants in AfricanAmerican women. PLoS ONE. 2013;8:e58350.

28. Purrington KS, Slager S, Eccles D, et al. Genome-wide association study identifies 25 known breast cancer susceptibility loci as risk factors for triple-negative breast cancer. Carcinogenesis. 2014;35:1012-19.

29. Buys SS, Sandbach JF, Gammon A, et al. A study of over 35,000 women with breast cancer tested with a 25 -gene panel of hereditary cancer genes. Cancer. 2017;123:1721-30.

30. Lakhani SR, Manek S, Penault-Llorca F, et al. Pathology of ovarian cancers in BRCA1 and BRCA2 carriers. Clin Cancer Res. 2004;10:2473-81.

31. Gevensleben H, Bossung V, Meindl A, et al. Pathological features of breast and ovarian cancers in RAD51C germline mutation carriers. Virchows Arch Int J Pathol. 2014;465:365-69.

32. Figueroa JD, Garcia-Closas M, Humphreys M, et al. Associations of common variants at $1 \mathrm{p} 11.2$ and $14 \mathrm{q} 24.1$ (RAD51L1) with breast cancer risk and heterogeneity by tumor subtype: findings from the breast cancer association consortium. Hum Mol Genet. 2011;20:4693-06.

33. Stevens KN, Vachon CM, Lee AM, et al. Common breast cancer susceptibility loci are associated with triple-negative breast cancer. Cancer Res. 2011;71:6240-49.

34. Thomas G, Jacobs KB, Kraft P, et al. A multistage genome-wide association study in breast cancer identifies two new risk alleles at 1p11.2 and 14q24.1 (RAD51L1). Nat Genet. 2009;41:579-84.

35. Orr N, Lemnrau A, Cooke R, et al. Genome-wide association study identifies a common variant in RAD51B associated with male breast cancer risk. Nat Genet. 2012;44:1182-84.

36. Osorio A, Endt D, Fernández F, et al. Predominance of pathogenic missense variants in the RAD51C gene occurring in breast and ovarian cancer families. Hum Mol Genet. 2012;21:2889-98.

37. Coulet F, Fajac A, Colas C, et al. Germline RAD51C mutations in ovarian cancer susceptibility. Clin Genet. 2013;83:332-36.

38. Thompson ER, Boyle SE, Johnson J, et al. Analysis of RAD51C germline mutations in high-risk breast and ovarian cancer families and ovarian cancer patients. Hum Mutat. 2012;33:95-99.

39. Blanco A, Gutiérrez-Enríquez S, Santamariña M, et al. RAD51C germline mutations found in Spanish site-specific breast cancer and breast-ovarian cancer families. Breast Cancer Res Treat. 2014;147:133-43.

40. Schnurbein G, Hauke J, Wappenschmidt B, et al. RAD51C deletion screening identifies a recurrent gross deletion in breast cancer and ovarian cancer families. Breast Cancer Res. 2013;15: R120.

41. Vuorela M, Pylkäs K, Hartikainen JM, et al. Further evidence for the contribution of the RAD51C gene in hereditary breast and ovarian cancer susceptibility. Breast Cancer Res Treat. 2011;130:1003-10.

42. Gutiérrez-Enríquez S, Bonache S, de Garibay GR, et al. About $1 \%$ of the breast and ovarian Spanish families testing negative for BRCA1 and BRCA2 are carriers of RAD51D pathogenic variants. Int J Cancer. 2014;134:2088-97.

43. Osher DJ, De Leeneer K, Michils G, et al. Mutation analysis of RAD51D in non-BRCA1/2 ovarian and breast cancer families. $\mathrm{Br}$ J Cancer. 2012;106:1460-63.

44. Baker JL, Schwab RB, Wallace AM, Madlensky L. Breast cancer in a RAD51D mutation carrier: case report and review of the literature. Clin Breast Cancer. 2015;15:e71-75.

45. He Y, Zhang Y, Jin C, et al. Impact of XRCC2 Arg188His polymorphism on cancer susceptibility: a meta-analysis. PLoS ONE. 2014;9:e91202.

46. Zhai M, Wang Y, Jiang MF Arg188His polymorphism in the $\mathrm{XRCC} 2$ gene and the risk of ovarian cancer: a meta-analysis. Genet Mol Res. 2015;14:10808-815.

47. Kamali M, Hamadani S, Neamatzadeh H, et al. Association of XRCC2rs3218536 polymorphism with susceptibility of breast and ovarian cancer: a systematic review and meta-analysis. Asian Pac J Cancer Prev. 2017;18:1743-749.

48. Sopik V, Akbari MR, Narod SA Genetic testing for RAD51C mutations: in the clinic and community. Clin Genet. 2015;88:303-12.

49. Drew $\mathrm{Y}$ The development of PARP inhibitors in ovarian cancer: from bench to bedside. Br J Cancer. 2015;113:S3-S9. 